\title{
La conservación-restauración de bienes culturales y los desafíos a los que se enfrenta
}

\author{
Pilar Aguilar Solves | coordinadora de difusión de la Asociación Profesional de Conservadores-Restauradores de España \\ URL de la contribución <www.iaph.es/revistaph/index.php/revistaph/article/view/3542>
}

Ejecutada bajo un discreto anonimato y con la voluntad de respeto al original, la profesión del conservador-restaurador de bienes culturales y su regulación parecen temas olvidados en el momento de crisis económica actual. Sin olvidar la prioridad en las inversiones en sanidad y educación, los profesionales del patrimonio debemos recordar a la clase política y a la sociedad la importancia de dedicar recursos monetarios y de personal a la salvaguardia de los bienes culturales con el objetivo de garantizar su conservación otros tantos siglos. Se propone en este texto que cierra el debate en torno al futuro de la conservación-restauración de bienes culturales realizar un análisis del estado actual de la cuestión desde un punto de vista crítico.

El pasado 29 de julio desde el Grupo de Difusión de ACRE realizamos la actividad \#ACREopina, coordinada por Ángela Espinar, en la que se animaba a los usuarios de nuestro grupo en Facebook a exponer sus opiniones en torno a este debate que hemos generado en la revista ph. Desde el Grupo de ACRE en Linkedln también hemos desarrollado buenos debates en relación con todos los temas que preocupan a la profesión. De esta intensa interacción se han formularon una serie de preguntas acerca del futuro de la profesión que analizaré a continuación en relación a diferentes bloques temáticos.

\section{Las competencias básicas para el ejercicio de la profesión}

Según indica el documento de ECCO (Confederación Europea de Organizaciones de Conservadores-

Restauradores) Directrices profesionales de ECCO: la profesión y su código ético (DIRECTRICES, 2002) para mantener los estándares de la profesión de conservador-restaurador de bienes culturales, la educación de los mismo y su formación debe estar al nivel de un más- ter universitario o uno equivalente reconocido. Esto es un nivel 5 en el Catálogo Nacional de Cualificaciones Profesionales (CNCP) y un nivel 7 en el European Qualification Network (EQF).

En el Real Decreto 1027/2011 se establece el Marco Español de Cualificaciones para la Educación Superior (MECES), que es una pieza necesaria para completar el Marco Español de Cualificaciones (MECU). Ambos se relacionan con el marco europeo de cualificaciones (EQF). El alcance del MECES se ciñe a las titulaciones correspondientes a la educación superior. Corresponde en sus niveles a los niveles 5, 6, 7 y 8 del EQF, completando la correlación entre el MECU y el EQF.

La realidad de la profesión, sin embargo, no tiene nada que ver con la teoría anteriormente explicada. Dejando aparte el intrusismo profesional por parte no sólo de individuos con otras titulaciones sino también carentes de ellas, en las convocatorias de empleo público en España las titulaciones requeridas podían ser una o dos: diplomado por las escuelas de conservación-restauración de bienes culturales o licenciado en Bellas Artes con especialidad en la universidad. Esa duplicidad de titulaciones que capacitaban ambas para un mismo ejercicio profesional no hizo más que agudizar el distanciamiento entre las escuelas y las universidades.

Con la llegada del Plan Bolonia parecía que la unificación sería posible con la creación del grado en ConservaciónRestauración de Bienes Culturales. Bolonia divide la enseñanza superior en 3 niveles: El grado tiene una duración de 4 años, 240 créditos, y sustituye a las diplomaturas y licenciaturas. El máster supone una especialización en un ámbito concreto o multidisciplinar y puede tener una duración de 1 año (60 créditos) o 2 (120). 
a debate El futuro de la conservación-restauración de bienes culturales | coordina Pilar Aguilar Solves

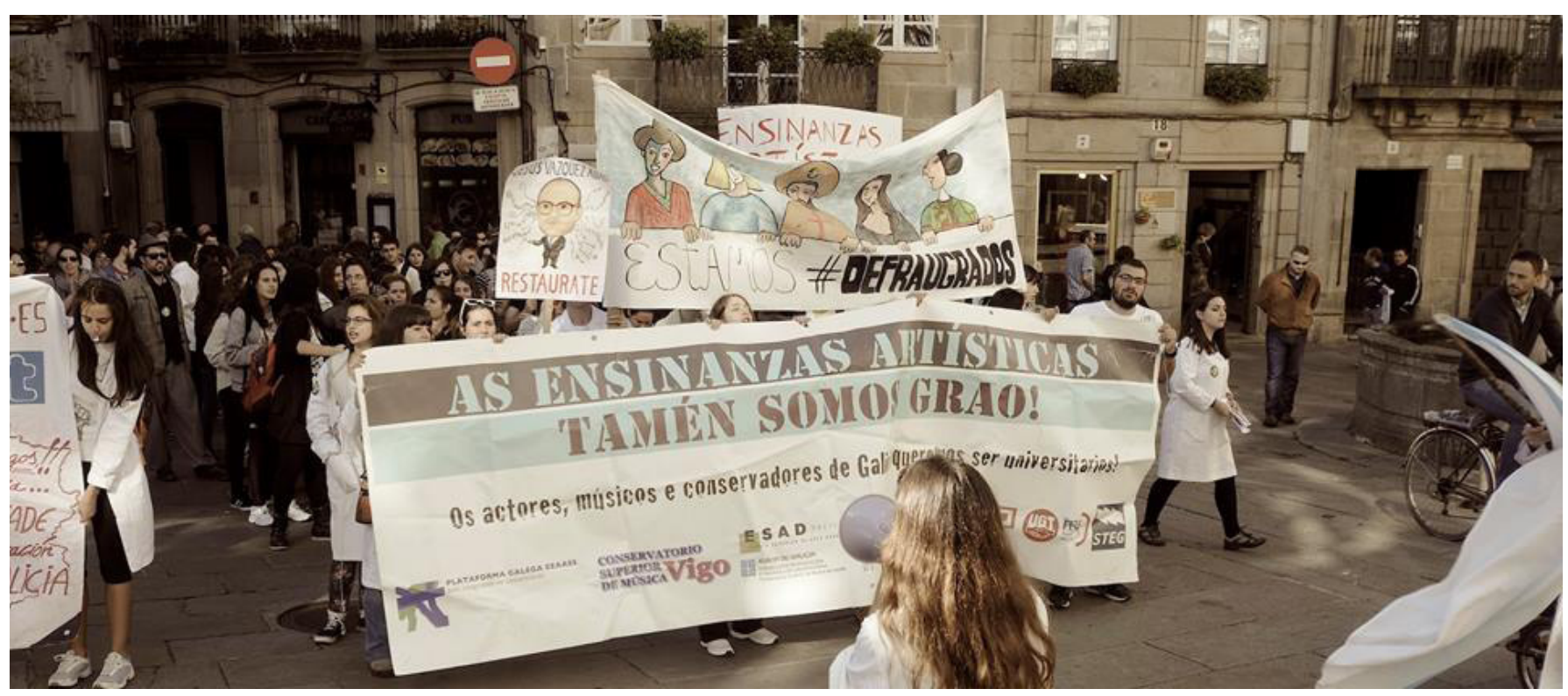

Movilizaciones de la Escuela Superior de Conservación-Restauración de Galicia con la marcha a Santiago de Compostela para pedir la adscripción de los estudios superiores de conservación-restauración a la Universidad. 8 de octubre de 2013 | foto ACRE

Sin embargo, el Tribunal Supremo, en la Sala de lo Contencioso-Administrativo (Sección Cuarta) en febrero de 2012, estimó en parte la impugnación por parte de la Universidad de Granada del Real Decreto 1614/2009, de 26 de octubre, que estableció la ordenación de las enseñanzas artísticas superiores reguladas por la LOE 2/2006. En esta sentencia se anulaban los artículos del RD 1614/2009 que hacían mención a los títulos de grado, por ser estos exclusivos de las universidades, y la LOE contemplaba únicamente la denominación de título superior ( $y$, por tanto, no grado) para las enseñanzas artísticas superiores:

"Realizadas las consideraciones que anteceden, conviene subrayar que las enseñanzas artísticas superiores, aunque dan lugar a la obtención de un título equivalente al universitario de Grado y, en su caso, de
Máster o Doctor, no son enseñanzas propiamente universitarias, por cuanto no se imparten en centros universitarios. Aunque tanto las enseñanzas artísticas superiores como las enseñanzas universitarias forman parte de la educación superior, no son de la misma naturaleza. El deslinde entre unas y otras corresponde a una decisión del legislador, plasmada en la Ley Orgánica 2/2006, de 3 de mayo, de Educación, con la que el proyecto de Real Decreto es consecuente" (DOCUMENTO CE-D-2009-1409).

Es decir, se considera que los títulos de grado son exclusivos de las universidades, contemplando la denominación de titulado superior para las enseñanzas artísticas oficiales (música, danza, arte dramático, diseño y conservación-restauración de bienes culturales). 


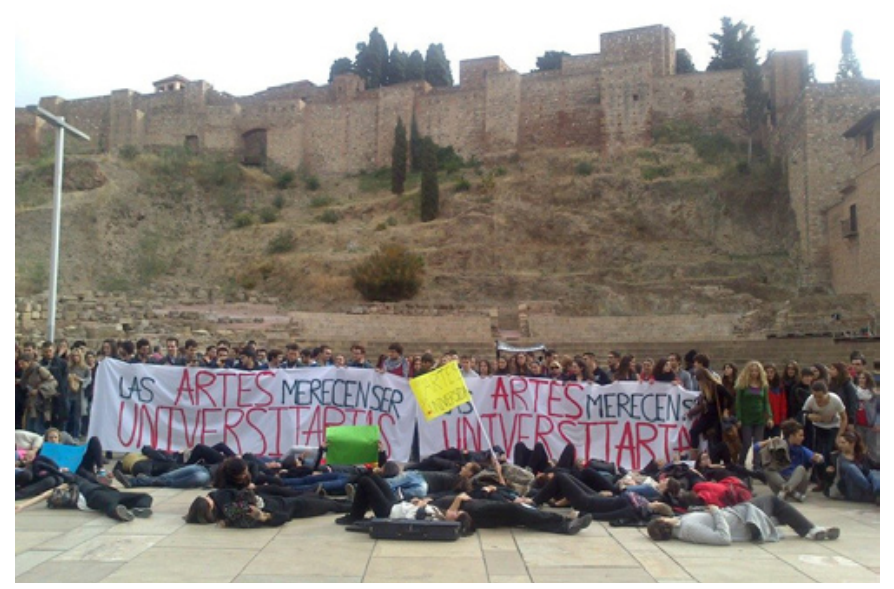

Marcha reivindicativa por la obtención del grado universitario de las enseñanzas artística. Málaga, diciembre de 2013 | foto La Taberna Global (Isabel Bellido)

En este momento es cuando se produce un baile de términos que origina confusiones: los títulos superiores no son universitarios, son equivalentes, mientras los grados sí que son universitarios. Al anular el Tribunal Supremo la denominación grado que se emplea en el Real Decreto 1614/2009 para referirse al título de las enseñanzas artísticas superiores, dejó en una situación de indefensión las expectativas de los alumnos que estaban matriculados en estas enseñanzas bajo esta denominación.

Esta nueva situación de re-duplicidad (títulos superiores de las escuelas que imparten las enseñanzas artísticas superiores versus grado de las universidades) ha provocado movimientos como los \#defraugrados iniciados desde la Escola Superior de Conservación e Restauración de Bens Culturais de Galicia, ejemplo de espíritu reivindicativo. Los alumnos de las escuelas demandan la adscripción a la universidad con el objetivo ser universitarios de pleno derecho ${ }^{1}$.

\section{La formación superior y la realidad profesional}

Somos muchas las personas que observamos la gran diferencia que hay entre los conocimientos teórico-prácticos que adquirimos en la facultad y el ejercicio de la actividad laboral. Debido probablemente a que una parte importante del profesorado está alejado del mundo laboral en el temario de las titulaciones no se imparten conocimientos sobre la gestión empresarial, la búsqueda de empleo, la elaboración de presupuestos,etc. El alumno una vez concluidos sus estudios se encuentra ante un terreno laboral en el que tendrá que integrarse y desconoce totalmente.

Los alumnos y exalumnos de las universidades españolas piden una respuesta enérgica de los rectores ante la elevada tasa de desempleo (el 50\% entre los jóvenes y el $25,6 \%$ en la totalidad de la población), la más alta de Europa.

La mayor implicación de la universidad en el terreno laboral es una demanda que no es sólo exclusiva de nuestro sector sino también de otros campos profesionales. Para la directora de Marketing y Comunicación de Adecco (empresa de colocación laboral) el problema fundamental es que la universidad pública desconoce la realidad empresarial y trabaja a pesar de ello formando mentes que no se ajustan a ella. En el estudio realizado desde su gabinete informa que el $47 \%$ de las empresas solicitan perfiles que la universidad no es capaz de producir (DIFÍCIL, 2014).

Me parece relevante también añadir que es tan importante preparar a los universitarios en el campo de trabajo como formarlos en valores éticos y humanos. La nueva dinámica de la sociedad demanda individuos no sólo con pensamiento crítico y formación ética para contribuir con el desarrollo sostenible, sino también con capacidades sólidas para sobrevivir en un mercado laboral cada vez más exigente.

\section{La importancia de la regulación del conservador- restaurador. La acreditación profesional europea}

La Unión Europea estableció en marzo de 2004 los Principios comunes europeos para la validación del aprendizaje no formal e informal, que son necesarios para fomentar la comparabilidad y la aceptación de la diferencias entre los Estados miembros así como para la 
a debate El futuro de la conservación-restauración de bienes culturales | coordina Pilar Aguilar Solves

transferencia y aceptación de los resultados educativos y formativos en distintos entornos. Las acreditaciones profesionales son un conjunto de actuaciones dirigidas a reconocer, evaluar y acreditar las competencias profesionales adquiridas mediante la experiencia laboral o de vías no formales de formación. Esta evaluación y acreditación de las competencias profesionales se desarrollará siguiendo criterios que garanticen la fiabilidad, objetividad y rigor técnico de la evaluación. El Catálogo Nacional de Cualificaciones Profesionales sirve de referencia objetiva en este procedimiento. El ámbito de la conservación de bienes culturales no está contemplado como tal en el Catálogo, dado que los criterios de actuación sobre piezas de valor patrimonial requieren un conocimiento más extenso que el simple procedimiento manual. Sin embargo, determinados procesos relacionados con la conservación-restauración aparecen en algunas cualificaciones como parte de un proceso auxiliar de colaboración (LÍMITES, 2013).

La profesión de conservador-restaurador en España no está regulada, como puede leerse en la respuesta de la Subdirección General de Aprendizaje a lo Largo de la Vida a la carta emitida por ACRE en la que informaba que el Curso Mentor de Conservación Restauración de Objetos Antiguos (promovido por el Ayuntamiento de Granada y el Ministerio de Educación Cultura y Deporte) no capacita para el ejercicio de la profesión:

"En el caso de las profesiones reguladas, y por expreso mandato de la ley, existe un título habilitante para dicho ejercicio de manera que solo su posesión habilita para esa profesión, de forma tal que quien no lo tiene no puede ejercerla.

\section{[...]}

En este sentido, le participo que la determinación de las profesiones reguladas ha de hacerse por Ley, circunstancia que no se da en la profesión que representa, por lo que los títulos que usted menciona (títulos emitidos por las Escuelas Superiores de Conservación y Restauración de bienes culturales y Licenciaturas en Bellas Artes con la especialidad en Conservación y Restauración), son títulos que habilitan para el ejercicio de la profesión de conservador-restaurador, pero no excluyentes de cualquier otro que esté dotado de las competencias específicas" (RESPUESTA, 2013).

En Reino Unido el Institute of Conservation (ICON) otorga una acreditación profesional certificada de nivel superior: Professional Accreditation of Conservator-Restorers ${ }^{2}$ (PACR), y otra inferior llamada cualificación técnica: Conservation Techician Qualification. Una vez obtenida, el profesional debe ir actualizándose en formación

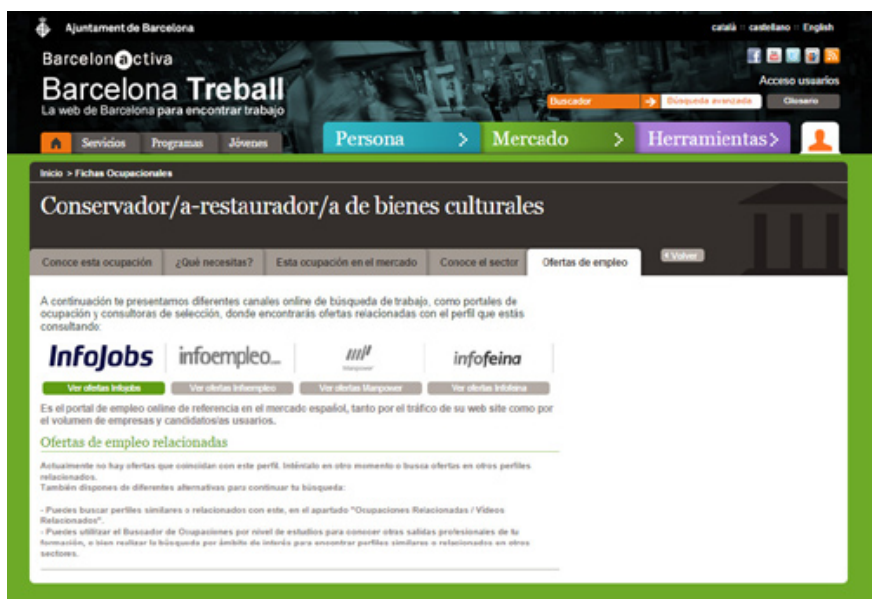

En España el empleo en conservación-restauración es prácticamente inexistente en los portales de trabajo

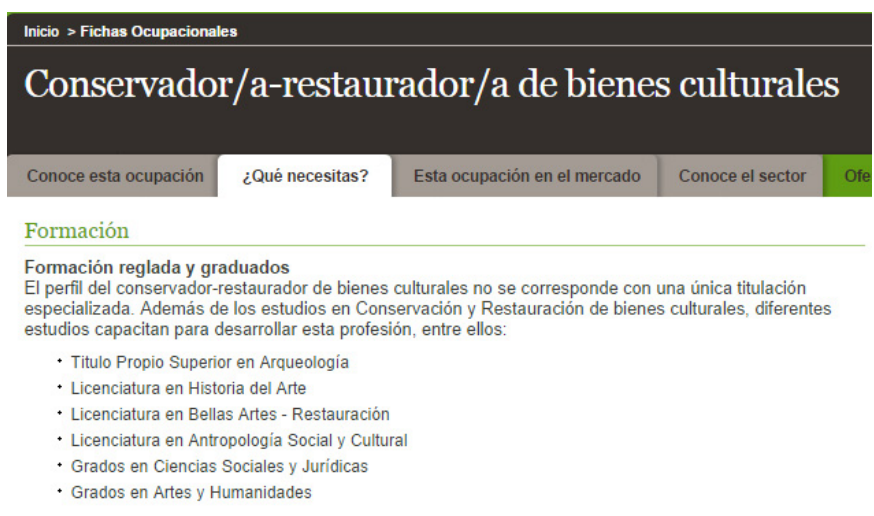

La formación que necesita un conservador-restaurador de bienes culturales para ejercer, según el Ayuntamiento de Barcelona. La falta de regulación profesional posibilita que se admitan diferentes titulaciones

fotos Barcelona Treball. La web de Barcelona para encontrar trabajo 
a debate El futuro de la conservación-restauración de bienes culturales | coordina Pilar Aguilar Solves

y capacidades: Continuing Professional Development (CPD). Estas acreditaciones no reservan de modo exclusivo el ejercicio de la profesión pero son muy prestigiosas profesionalmente, como explica en esta misma sección, María José Prieto Pedregal (pp. 160-163).

Si volvemos a España podemos encontrar (desgraciadamente) numerosos casos que evidencian las razones por las cuales es necesaria una regulación de la profesión. El primer ejemplo que seguro que todos recordamos es el conocido Ecce Homo de Borja (HUERTA, 2012). La fallida actuación de la octogenaria murciana repintando por completo la obra de Elías García Martínez fue motivo de burla internacional. En esos días realicé una entrevista para el diario de internet About. com (KENDZULAK, 2014) en el que me preguntaba conmocionada cómo había afectado este caso a España y qué estaba haciendo el país para intentar evitar eso. La respuesta era es sencilla y sigue siendo la misma: nada. La ausencia de una regulación profesional que acabe con el intrusismo y una legislación para los bienes culturales más exigente que penalice ese tipo de actuaciones sitúan al patrimonio español en una situación de permanente indefensión.

\section{La labor de los colegios y las asociaciones profesionales}

En España hay pocas asociaciones en funcionamiento, sólo una a nivel nacional: la Asociación Profesional de Conservadores-Restauradores de España, $\mathrm{ACRE}^{3}$. Las asociaciones nos dan el reconocimiento de los organismos estatales y de la sociedad en general; ayudan a que se aprueben leyes nacionales que protejan nuestra profesión y el patrimonio cultural; establecen los parámetros para acceder a la profesión; elaboran criterios a nivel nacional e internacional que representan nuestros intereses y no los de otras profesiones con las que solemos estar asociados, como arquitectos y constructores (BORDALO, 2011).

Sobre la existencia de otras figuras profesionales relacionadas con la del conservador-restaurador
Actualmente en Italia se está desarrollando el proceso para reconocer la profesión de conservador-restaurador de bienes culturales y la de ayudante de conservadorrestaurador. Con el registro del Tribunal de Cuentas, el 6 de agosto de 2014, la Orden Ministerial de 13 de mayo 2014 aprobó la aplicación Directrices del artículo 182 del Código del Patrimonio Cultural y Paisaje, se inicia el procedimiento previsto en el régimen transitorio (Ley n. ${ }^{\circ}$, de 14 de enero de 2013) para el logro de las cualificaciones profesionales como la de restaurador de bienes culturales y el asistente del restaurador de bienes culturales.

\section{Cómo dar visibilidad a nuestro trabajo}

En los últimos años las instituciones culturales están realizando proyectos en los que los restauradores trabajan fuera de su lugar habitual y restauran frente al público. Este es el caso de El Paraíso de Tintoretto, en el Museo Thyssen-Bornemisza en Madrid, o La crucifixión de San Andrés de Caravaggio, en el Cleveland Museum of Art en Ohio.

Desde siempre la conservación-restauración de bienes culturales ha sido una profesión admirada por la gente que ve en ella un pequeño "milagro", cosa que interesa pues nos ayuda a mantener su carácter misterioso.

La restauración en muchas ocasiones es un trabajo realizado a puerta abierta donde el espacio es invadido por el público usuario de las instalaciones.

Para algunos restauradores este tipo de iniciativas tienen más de espectáculo que de voluntad informativa. Sin embargo, para otros este tipo de acciones son una nueva forma de dar a conocer la profesión, de mostrar cómo es nuestro trabajo.

Ésta es una de las maneras de dar visibilidad al ejercicio profesional pero también hay otras como la participación en revistas de impacto científico, la creación de talleres infantiles y la participación en congresos más allá de nuestra disciplina. El objetivo del conservador-restaurador del siglo XXI tiene que ser el dar a conocer su trabajo 
a través de las nuevas tecnologías de la información y de las redes sociales acercando su día a día a espectadores de todo tipo y de todas partes del mundo.

\section{El mercado laboral}

Según el estudio realizado por el Ministerio de Educación, Cultura y Deporte sobre el sector de los profesionales de museos (PROFESIONALES, 2012) en el que aparece la figura del conservador-restaurador de bienes culturales, la mayoría de estos (más del 80\%) no tiene expectativas de mejorar en su puesto de trabajo, ni en responsabilidad ni en remuneración durante los próximos tres años. Los trabajadores de museos de titularidad pública son menos optimistas que los que lo hacen en la privada. Si entre los profesionales del sector público la crisis económica genera un fuerte pesimismo, no lo es menos en aquellos profesionales que trabajan por cuenta propia y fuera del sector de museos.

Según el informe del 2014 realizado por Deloitte para el Ayuntamiento de Barcelona, la conservación-restauración de bienes culturales ocupa el séptimo puesto entre las profesiones con mayor cualificación requerida entre las más demandadas del sector cultural (OCUPACIONS, 2014). Se encuentra en el penúltimo puesto por encima de los coordinadores de exposiciones. Entre los datos que poseemos en la Asociación Profesional de Conservadores-Restauradores de España, llama la atención la alta formación (la mayoría con dos titulaciones y máster). Buena parte de nuestros seguidores desde los 23 años se encuentra en situación de desempleo; aquellos que sí trabajan en el sector cultural lo hacen bajo la fórmula de contrato temporal o beca. Todos los trabajadores (independientemente de que sigan ejerciendo o no la profesión) consideran que su categoría laboral es inferior a la que les corresponde. Entre aquellos con edades comprendidas entre los 40-60 encontramos tres tipos de profesionales: los que trabajan en instituciones públicas (docentes, museos, centros de restauración...) y los que trabajan por cuenta propia. De estos últimos muchos han visto ya sus empresas extintas y se emplean bajo la fórmula del profesional autónomo.

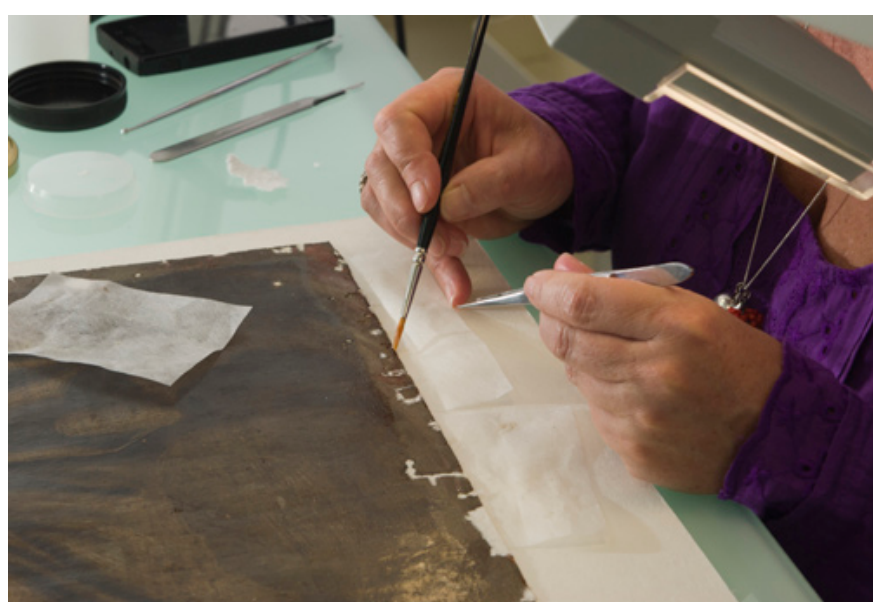

Taller de papel. Centro de Intervención, IAPH | foto Fondo Gráfico IAPH (Eugenio Fernández)

Los resultados de la encuesta realizada por el Ministerio de Educación, Cultura y Deporte anteriormente mencionada muestran la importancia de la formación complementaria entre los profesionales de los museos y cómo ha aumentado la tendencia a realizar cursos específicos en museos y patrimonio entre los profesionales incorporados recientemente.

Queda claro que es relevante que los profesionales actualicen los conocimientos a través no sólo de los cursos promovidos desde los centros académicos tradicionales (escuelas, universidades e institutos de restauración) sino también desde los servicios de formación públicos.

Desde que en 2009 creé el Blog Quermes ${ }^{4}$ (el más leído en España en el sector de la conservación-restauración) y en 2012 asumiera la coordinación del Grupo de Difusión de la Asociación Profesional de ConservadoresRestauradores de España, han sido miles las publicaciones que hemos realizado en las redes sociales y otros tantos los mensajes a los que hemos respondido. A pesar de nuestra gran capacidad de difusión, creación y dinamización como generadores de opinión, tras estos años de experiencia puedo afirmar que se palpa cierta falta de implicación personal de los profesionales del sector. Basándome en esta experiencia personal puedo afirmar que esto se debe a varios factores: El primero, la ausencia de conexión entre la formación académica y el ejer- 
a debate El futuro de la conservación-restauración de bienes culturales | coordina Pilar Aguilar Solves

cicio profesional real; el segundo, la precariedad laboral del sector que siempre nos ha acompañado. La crisis económica no ha hecho más que agudizar el segundo punto.

Resulta curioso que en una profesión en la que tanto se habla de interdisciplinariedad y en la que se trabaja con profesionales de diferentes sectores, no haya reivindicado como colectivo todo lo que le corresponde y se haya movilizado de forma masiva. Los conservadores-restauradores parece que no sepamos qué hacer y hayamos perdido toda esperanza, nos encontramos pues, rendidos ante la situación aceptando lo bueno y lo malo que viene con ella. En un momento en el que se está produciendo un cambio social intenso y extenso donde se cuestionan las bases de nuestra civilización y se plantean nuevos horizontes construidos desde cero, creo que los conservadores-restauradores tenemos que aprender a reconciliar las limitaciones que nos impone el presente y luchar por llegar lo más lejos posible. "No puedes cambiar el viento, pero puedes cambiar la dirección de tus velas", reza un proverbio chino.

\section{NOTAS}

1. Plataforma por la integración de las Enseñanzas Artísticas Superiores en el Sistema Universitario <http://www.eeaassuniversidad.guidoblogs.org/>.

2. ICON. Accreditation (PACR) and Professional Standards <http:// www.icon.org.uk/index.php?option=com_content\&task=view\&id=5\&lte mid $=6 />$.

3. Asociación Profesional de Conservadores-Restauradores de España, ACRE <http://www.asociacion-acre.com>.

4. Blog Quermes: <http://www.pilaraguilar.com>; <http://www.facebook.com/BlogQuermes>. 
_a debate El futuro de la conservación-restauración de bienes culturales | coordina Pilar Aguilar Solves

\section{BIBLIOGRAFÍA}

- Bordalo, R. (2011) The Worthiness of Association. econservation [en línea], n. ${ }^{\circ} 20$, July 2011 , p. 4 <http://www. e-conservationline.com/content/view/1003> [consulta: 15/09/ 2014]

- DIFícIL (2014) ¿Por qué es tan difícil el paso de la universidad al mercado laboral? En universia [en línea]. España: noticias de actualidad, 27/02/2014 <http://noticias. universia.es/en-portada/noticia/2014/02/27/1084296/que-estan-dificil-paso-universidad-mercado-laboral.html> [consulta: 15/09/2014]

- DIRECTRICES (2002) Directrices profesionales de ECCO: la profesión y su código ético. En Grupo Español de Conservación del IIC. Cartas y documentos [en línea]. 1 de marzo de 2002 $<$ http://ge-iic.com/files/Cartasydocumentos/2002_directrices_ \%20profesionales_de_ecco_la_profesion_y_su_codigo_etico. pdf> [consulta: 15/09/2014]

- DOCUMENTO CE-D-2009-1409. Dictamen del Consejo de Estado sobre el Proyecto de Real Decreto por el que se establece la ordenación de las enseñanzas artísticas superiores reguladas por la Ley Orgánica 2/2006. En Agencia Estatal Boletín Oficial del Estado: Dictámenes del Consejo de Estado [en línea] <http://www.boe.es/buscar/doc.php?id=CED-2009-1409> [consulta: 15/09/2014]

- HERNÁNDEZ REVUELTA, A. (2012) Enseñanzas Artísticas. Situación en España y en Europa: oferta académica [en línea]. Madrid: Universidad Complutense de Madrid, 2012 <https:// bellasartes.ucm.es/data/cont/media/www/pag-11069/ense\%C 3\%B1anzas\%20artisticas.pdf> [consulta: 15/09/2014]

- HUERTA, R. (2012) La restauración de un eccehomo se convierte en un sainete mundial. El País [en línea], Cultura, 23 agosto 2012 <http://cultura.elpais.com/cultura/2012/08/23/ actualidad/1345709139_149007.html> [consulta: 15/09/2014]

- KENDZULAK, S. (2014). Q and A with art conservator Pilar Aguilar. How to stablish your own career. About.com [en línea] About careers <http://fineart.about.com/od/FineArt-Restoration/a/Q-And-A-With-Art-Conservator-PilarAguilar-On-How-To-Establish-Your-Art-Career.htm> [consulta: 15/09/2014]

- LímITES (2013) Los límites de la formación profesional. En Asociación de Conservadores Restauradores de España [en línea]. Septiembre, 2013 <http://es.scribd.com/ doc/166509263/Los-limites-de-la-formacion-profesional> [consulta: 15/09/2014]

- MURCIA MOLINA, S. (2013). La actual inseguridad jurídica de las enseñanzas artísticas. Actores 108: revista de la Unión de Actores, primavera 2013 [en línea] <http://www. revistaactores.com/ua_revista/n21/web/teinteresa_12.html > [consulta: 15/09/2014]
- OCUPACIONS (2014) Ocupacions més demandades al sector de la Cultura. Informe 2014 [Barcelona]: Ayuntament, $2014<$ http://w27.bcn.cat/porta22/images/es/Barcelona_Treball _Ocupacions_Cultura_cat_tcm24-36709.pdf> [consulta: 15/09 /2014]

- PRIETO, M.; CASAnOVA, M. (2013). Los límites de la formación profesional. En Asociación de Conservadores Restauradores de España [en línea] <http://es.scribd.com/ doc/166509263/Los-limites-de-la-formacion-profesional> [consulta: 15/09/2014]

- PROFEsionAles (2012) Los profesionales de los museos. Un estudio sobre el sector en España [en línea]. [Madrid]: Secretaría General Técnica. Centro de Publicaciones. Ministerio de Educación, Cultura y Deporte, 2012, 128 p. <https://sede. educacion.gob.es/publiventa/detalle. action?cod=14316C> [consulta: 15/09/2014]

- RESPUESTA (2013) Respuesta Subdirección General de Aprendizaje a lo largo de la vida. En Asociación de Conservadores Restauradores de España. Abril, 2013 < http:// es.scribd.com/doc/155742671/Respuesta-SubdireccionGeneral-de-Aprendizaje-a-lo-largo-de-la-vida> [consulta: 15/ 09/2014]

- SISTEMA (2014) El sistema universitario español y el Espacio Europeo de Educación Superior. En Universidad de Córdoba. Espacio Europeo de Educación Superior. Normas y Documentos [en línea] <http://www.uco.es/organizacion/eees/ documentos/normas-documentos/otros/EI\%20Sistema\%20U niversitario\%20Espanol\%20y\%20el\%20EEES.pdf> [consulta: 15/09/2014]

- ST 96002004 INIT (2004) ST 96002004 INIT. Proyecto de Conclusiones del Consejo y de los Representantes de los Gobiernos de los Estados miembros reunidos en el seno del Consejo sobre los Principios europeos comunes para la determinación y convalidación de la educación no formal e informal . En Consejo de la Unión Europea. Documentos [en línea] <http://register.consilium.europa.eu/doc/srv?l=ES\&f=ST \%209600\%202004\%20INIT> [consulta: 15/09/2014] 